\title{
Collaborer pour développer des pratiques d'enseignement de science et technologie à l'école primaire : des retombées pour la pratique, la recherche et la formation
}

Par Christine Couture, Université du Québec à Chicoutimi

Emmanuelle Aurousseau, Université du Québec à Chicoutimi

Yvan Lévesque, Commission scolaire des Rives-du-Saguenay

Pauline Tremblay, Commission scolaire De La Jonquière

Avec la collaboration d'enseignants des commissions scolaires De La Jonquière et des Rives-du-Saguenay et avec le soutien financier du Consortium régional de recherche en éducation de l'UQAC (CRRE, 20052007), du Ministère du Développement Économique de l'Innovation (MDEI, 2005-2007) et du Conseil de recherche en sciences humaines (CRSH, 2008-2011; 2013-2017)

\section{Résumé}

Le développement des pratiques d'enseignement est un processus long et complexe pouvant se faire dans différents contextes. Pour soutenir un tel développement, la collaboration entre différents acteurs est souvent mise de l'avant dans des perspectives allant de la formation continue à la recherche. La question des retombées de tout ce travail de collaboration demeure toutefois difficile à saisir. Afin d'apporter un éclairage sur cette question, ce texte propose une réflexion sur les retombées de trois projets de recherche collaborative menés avec des enseignants du Saguenay, de 2004 à 2016, pour développer et documenter des pratiques d'enseignement de science et technologie au primaire. Pour ce faire, une mise en contexte présente l'évolution de ces projets, quelques balises théoriques en donnent les orientations et les considérations méthodologiques en décrivent le déroulement. Les constats sont ensuite présentés en termes de retombées pour la pratique, la recherche et la formation.

Mots-clés : Recherche collaborative, développement de pratiques, science et technologie, enseignement primaire 


\section{REVUE HYBRIDE DE L'ÉDUCATION}

\section{Introduction}

En éducation, la recherche collaborative est depuis les années 1990 reconnue pour son intention de réduire l'écart entre le monde de la recherche et le monde de la pratique (Desgagné, Bednarz, Couture, Poirier et Lebuis, 2001). Cette idée de rapprochement, voire de partage d'expertise, a inspiré de nombreux projets ayant pris des formes diverses et ayant généré des résultats variés. Que reste-t-il de tous ces projets où praticiens et chercheurs ont travaillé ensemble à explorer de nouvelles perspectives? Une réponse précise à cette question serait bien fragile puisqu'au delà de ce que nos outils méthodologiques nous permettent de saisir, reste tout ce qui demeure plus ou moins formel en termes de retombées. C'est pour cette raison que ce texte propose une réflexion sur les retombées plus ou moins formelles d'un travail mené en collaboration avec des enseignants du Saguenay sur le développement de pratiques d'enseignement de science et technologie depuis un peu plus de dix ans (Couture, 2010, 2013, 2015; Couture, Dionne, Savoie-Zajc et Aurousseau, 2012, 2015).

\section{Mise en contexte}

Depuis 2004, nous avons eu l'occasion de travailler, avec des partenaires du milieu scolaire du Saguenay, à développer des pratiques d'enseignement de science et technologie au primaire dans le cadre de trois projets. Le premier (2004-2007), réalisé à la commission scolaire De La Jonquière et supporté par le Consortium régional de recherche en éducation (CRRE) et le Ministère du Développement Économique de I'Innovation (MDEI), a permis d'accompagner 27 enseignants de trois écoles différentes selon diverses modalités (travail en équipe-cycle, soutien individualisé, projets d'école). Le second (2008-2011) et le troisième (2013-2017), réalisés à la commission scolaire des Rives-duSaguenay et subventionnés par le Conseil de recherche en sciences humaines du Canada (CRSH), ont engagé 15 enseignants dans des communautés d'apprentissage pour développer des pratiques 


\section{REVUE HYBRIDE DE L'ÉDUCATION}

d'enseignement sur des périodes assez longues (près de deux ans) pour vivre des boucles itératives d'exploration, de planification conjointe, de réalisation en classe et de réflexion sur l'action (Couture, 2002).

En guise de retombées plus formelles, des publications professionnelles et scientifiques sur des ajustements et des exemples de pratique développés avec des enseignants (Couture 2010, 2013, 2015; Couture, Dionne, Savoie-Zajc et Aurousseau, 2012, 2015) sont disponibles. Au-delà de ces publications, une panoplie d'idées partagées et d'outils développés conjointement, de réflexion collective et de réinvestissements plus ou moins connus traversent nos projets. Une analyse rigoureuse de telles retombées est difficile à mener d'autant plus que les réinvestissements se font rarement à court terme. Pourtant, des retours parfois inattendus, au gré des rencontres, nous laissent croire que tout ce travail a porté fruit. Sans prétendre répondre aux critères de scientificité exigés par la recherche, une réflexion sur ces retombées moins formelles peut apporter un éclairage différent contribuant à documenter le potentiel peut-être moins visible de ce que nous faisons ensemble. Nous tentons toutefois de faire cette réflexion dans un cadre qui montre comment recherche et pratique peuvent s'influencer mutuellement.

\section{Quelques balises théoriques}

Les principales balises théoriques ayant guidé le travail de développement mené avec les enseignants concernent la collaboration praticiens-chercheurs, la communauté d'apprentissage, la pratique abordée dans une perspective d'ajustement plutôt que de changement et la didactique des sciences. Globalement, les idées fortes guidant la réalisation des projets évoqués dans ce texte pour chacun de ces axes se résument comme suit: La collaboration est d'abord une question de reconnaissance, de part et d'autre. En effet, pour collaborer, il faut non seulement avoir quelque chose à partager, mais aussi à recevoir. Le simple fait de travailler avec des enseignants ne suffit pas pour faire de la 


\section{REVUE HYBRIDE DE L'ÉDUCATION}

recherche collaborative. La recherche collaborative suppose la coconstruction de connaissances sur la pratique. L'apport des enseignants pour identifier ces connaissances, les questionner, les situer concrètement, les recadrer et les opérationnaliser est essentiel dans le processus. Selon Desgagné (1997, p.377), l'intérêt de cette approche réside dans « la croyance que la production de connaissances améliore la pratique et que la pratique éclaire la production de connaissances ». Dans le prolongement de cette idée, des ouvrages en didactique des sciences (Hasni et Samson, 2011; Kahn, Hersant et Orange Ravachol 2010; Samson, Couture et Sylla, 2015) témoignent d'une coexistence de travaux portant sur des collaborations pour développer des pratiques et des recherches collaboratives qui contribuent à construire des savoirs sur ce développement de pratiques. Cette coexistence entre développement et recherche est révélatrice des liens qui se tissent entre la pratique et la recherche même si la question des retombées n'est pas toujours claire.

La communauté d'apprentissage offre un espace propice à la coconstruction de connaissances sur la pratique. Dans le cadre de nos travaux, elle est considérée en termes de dispositif de formation et de recherche collectif et flexible favorisant le développement de la pratique (Dionne et Couture, 2013; Dionne, Lemyre et Savoie-Zajc, 2010). Repris dans un processus de recherche collaborative, un tel dispositif permet de répondre au double besoin de formation des enseignants et de production de connaissances sur des pratiques qui intègrent différentes perspectives dont celles des programmes en vigueur, de la recherche en didactique et de la pratique même des enseignants. La communauté d'apprentissage permet de mettre en place des activités réflexives (Desgagné, Bednarz, Couture, Poirier et Lebuis, 2001) contribuant tout autant au développement de la pratique qu'au développement de connaissances. Chacun bénéficie alors de l'expertise de l'autre, d'où l'idée de mener une réflexion plus approfondie sur les retombées d'un tel dispositif pour la pratique, la recherche et la formation. 


\section{REVUE HYBRIDE DE L'ÉDUCATION}

La pratique d'enseignement comprend un ensemble d'activités orienté par les savoirs et les compétences de l'enseignant, ainsi que par les normes de la profession (Legendre, 2005). Elle est contextualisée et orientée par la nature des apprentissages visés, ce qui interpelle les orientations proposées dans les programmes, mais aussi dans les travaux de recherche en didactique. Cette pratique est soumise à différentes sources d'influence dont les programmes, le matériel didactique, les projets d'école, le contexte de la classe et peut-être même la recherche. Dans les directives officielles, son développement est souvent présenté en termes de changement. Pourtant, un tel développement ne se fait pas nécessairement dans la rupture. II gagne même à y intégrer des éléments de continuité. C'est dans cette idée de continuité que le concept d'ajustement de pratique devient intéressant. II s'agit d'adaptations faites par les enseignants pour s'approcher d'un idéal à atteindre qu'ils dégagent d'une réflexion sur leurs propres pratiques (Savoie-Zajc, 2005). Dans notre travail avec les enseignants, cette réflexion est menée conjointement à partir des programmes et des travaux de recherche en didactique des sciences. C'est ainsi que la communauté d'apprentissage définit collectivement un idéal à atteindre tout en laissant à chaque enseignant la liberté de le déployer à sa façon. Des adaptations peuvent alors être pensées et développées sans rompre avec ce qui se fait déjà. C'est à travers ces adaptations que des exemples de pratique contribuant à documenter ce qui se développe collectivement prennent forme. De tels exemples présentent un potentiel à explorer en termes de retombées pour la pratique, la recherche et la formation en didactique.

La didactique des sciences ne relève pas que de la recherche. C'est aussi une question de programme et de choix des enseignants, ce qui rappelle les trois registres didactiques proposés par Martinand au début des années 1990. Martinand (1992) proposait alors une didactique critique et prospective (principalement liée à la recherche), une didactique normative (inhérente au programme) et une didactique praticienne (relative aux choix des enseignants) pour mettre en évidence la spécificité 


\section{8}

\section{REVUE HYBRIDE DE L'ÉDUCATION}

de chacune de ces perspectives et montrer l'intérêt de les mettre en dialogue. En plus de permettre d'intégrer des considérations venant de la pratique des enseignants, des programmes et des travaux de recherche, cette mise en dialogue rejoint l'idée de co-construction de connaissances sur la pratique qui est centrale en recherche collaborative. C'est alors que peut s'installer un véritable jeu d'influence réciproque (Bednarz, 2013; Desgagné 1997) basé sur la complémentarité des acteurs plutôt que sur la légitimité d'un point sur un autre. Dans nos travaux, ce jeu d'influence est à la base des ajustements et des exemples de pratique que nous développons avec les enseignants dans une perspective de didactique plus intégrative. Orientée vers l'action, une didactique plus intégrative met à contribution des regards multiples pour enrichir des situations d'enseignement et d'apprentissage prenant racine dans la pratique des enseignants, en y intégrant des éléments du programme et des travaux de recherche en didactique des sciences (Couture, 2013, 2015).

Pour guider le travail de développement réalisé conjointement, un certain nombre de balises issues des programmes et des travaux de recherche en didactique sont nécessaires. II importe de préciser que ces balises deviennent des grilles d'analyse pour réfléchir et développer collectivement et non pas des modèles à implanter. Ces orientations permettent ainsi de reconnaitre les éléments forts de la pratique, de questionner certains choix et de développer des ajustements en fonction d'un idéal visé collectivement. Afin de choisir ces balises, une synthèse d'orientations proposées dans les programmes de différents pays (Canada, États-Unis, France, Australie) et des travaux de recherche en didactique a été réalisée. Le détail de ce travail est disponible dans la revue française de Recherche en Didactique des Sciences et des Technologies (Couture, Dionne, Savoie-Zajc et Aurousseau, 2015). De cette synthèse, six critères ont été retenus pour guider notre travail de développement des pratiques d'enseignement de science et technologie. Pour l'enseignement de science et technologie, ces critères se présentent comme suit : 


\section{REVUE HYBRIDE DE L'ÉDUCATION}

- Susciter le questionnement;

- Engager l'élève dans des démarches d'investigation riches et variées;

- Établir des liens avec des problématiques sociales;

- Favoriser l'enrichissement conceptuel en mobilisant différentes formes de langage utilisées en sciences et technologies;

- Intégrer des technologies de l'information et des communications dans un processus de construction de connaissances;

- Intégrer autant les démarches que les connaissances dans l'évaluation des compétences.

(Couture, Dionne, Savoie-Zajc et Aurousseau, 2015, p. 122)

Les critères que nous avons retenus de notre recension des écrits ne représentent qu'une partie de notre cadre de réflexion qui s'enrichit des critères des enseignants pour expliquer leurs choix pédagogiques et didactiques en science et technologie. Ce double éclairage aide à définir collectivement notre idéal à atteindre en y intégrant ce qui se fait déjà. C'est dans cette idée de maillage de points de vue et d'expertises (Proulx, 2013) que le travail de développement et de recherche peut se faire dans un esprit de collaboration praticiens-chercheurs.

\section{Considérations méthodologiques}

La réflexion que nous menons nous amène à décrire globalement, sur le plan méthodologique, deux composantes essentielles dans la réalisation de toute recherche collaborative : le travail de terrain mené conjointement avec les acteurs et le travail de collecte et d'analyse de données. Notons d'abord que la recherche collaborative se distingue de la recherche-action par le rôle des acteurs dans chacune de ces approches. La recherche-action place les enseignants dans une posture de co-chercheurs (Savoie-Zajc, 2001), engagés dans différentes tâches liées à la recherche, alors que la recherche collaborative place plutôt les enseignants dans un rôle d'acteurs compétents (Desgagné, 1998) dont l'expertise est essentielle pour éclairer l'objet d'étude. Dans ce rôle 


\section{$\&$}

\section{REVUE HYBRIDE DE L'ÉDUCATION}

d'acteur compétent, l'enseignant est invité à mener, avec le chercheur, une réflexion sur la pratique s'inscrivant dans un processus de développement qui devient alors lieu de collecte et d'analyse de données. C'est ainsi que se mettent en place, dans le cadre de nos projets, des boucles itératives de planification conjointe, de réalisation en classe et d'analyse réflexive (Couture, 2002).

Les modalités de travail collectif sont multiples en recherche collaborative et gagnent à s'adapter aux demandes du milieu. D'ailleurs, plusieurs de ces demandes sont exprimées en termes de formation, ce qui pose le défi de l'arrimage avec la recherche puisque toute activité de formation ne génère pas nécessairement une activité de recherche. Dans nos projets, ces modalités ont pris la forme d'accompagnement d'équipescycles (Couture 2010, 2013; Couture, Dionne, Savoie-Zajc et Aurousseau, 2012) et de communautés d'apprentissage (Couture, Dionne, Savoie-Zajc et Aurousseau 2015; Dionne et Couture, 2013; Dionne, Couture, Savoie-Zajc et Paris, 2014) selon les demandes des milieux, les projets de recherche et les ressources disponibles pour mener ces projets. Avec l'aide des conseillers pédagogiques et avec de petits groupes d'enseignants, nous travaillons à décrire ce qui se fait déjà dans les classes en science et technologie, à l'analyser à la lumière de différents critères, à identifier les points forts et les aspects de la pratique à développer, à explorer différentes ressources, à planifier conjointement, à faire des essais en classe pour y revenir collectivement dans un esprit d'analyse réflexive. Ce travail intègre naturellement les projets en développement dans les milieux, dont les activités proposées par des organismes voués à la promotion de la culture scientifique. C'est ainsi que s'engage le dialogue entre pratique et recherche dans un processus qui permet de se connaitre, se reconnaitre et surtout de co-construire des connaissances riches de différentes perspectives.

Pour dégager des connaissances de notre travail collectif, encore faut-il saisir ce qui s'y passe. Dans une perspective de recherche qualitative interprétative permettant de saisir le sens de l'expérience 


\section{REVUE HYBRIDE DE L'ÉDUCATION}

(Savoie-Zajc, 2011), des compte-rendu de rencontres et des enregistrements audio ont permis de garder des traces de ce qui s'est construit conjointement. Des exemples précis de cette co-construction ont déjà été présentés dans d'autres textes en lien avec les ajustements de pratique (Couture, 2010; 2013). Ces exemples concernent des activités portant sur les mélanges, les réactions chimiques, les volcans, le système solaire, le cycle de l'eau, la construction de filtres à eau, d'anémomètres, de tours et de ponts. Une analyse de ces exemples par catégories conceptualisantes (Paillé et Muchielli, 2008) a aussi permis d'identifier des axes plus généraux de développement des pratiques d'enseignement (Couture 2015) liées à l'expérience, à la construction et à l'utilisation de modèles, aux démarches d'investigation ainsi qu'à la planification des apprentissages en science et technologie. Dans notre dernier projet (Couture, Dionne, Savoie-Zajc et Aurousseau, 2015), des réalisations d'élèves et des photos prises en classe s'ajoutent à la collecte de données pour formaliser, analyser et diffuser des exemples de pratique que nous construisons avec les enseignants.

\section{Réflexion sur les retombées pour la pratique, la recherche et la formation}

Trois axes sont retenus pour réfléchir aux retombées de nos travaux de recherche pour développer et documenter des pratiques d'enseignement de science et technologie : la pratique, la recherche et la formation.

\section{Retombées pour la pratique}

Les retombées de nos travaux pour la pratique demeurent difficiles à saisir à long terme, d'autant plus que le changement est un processus qui prend du temps. Lors de rencontres bilan, les enseignants ont témoigné de l'importance des moments de partage et d'échange et des prises de conscience relatives aux apprentissages visés en termes de processus plutôt que de résultats. Ils apprécient les propositions d'outils et d'activités à réaliser en classe, l'exploration collective de ressources didactiques 


\section{REVUE HYBRIDE DE L'ÉDUCATION}

ainsi que les moments de réflexion sur les pratiques. Mais les réinvestissements surviennent généralement plus tard, dans l'espace privé de la pratique, une fois la recherche terminée. C'est au gré des rencontres que l'un ou l'autre des enseignants nous raconte ce qu'il a développé dans le prolongement de nos échanges et comment le travail mené en collaboration a influencé ses choix. II serait périlleux d'essayer de mesurer de telles retombées plus subtiles et moins directes. Pour les rendre utiles dans la pratique, il est plus intéressant de les décrire en termes de pistes de développement et d'exemples de pratique. À cette fin, nous avons déjà dégagé quelques pistes de développement à proposer (Couture, 2015) à la lumière des ajustements de pratique déjà analysés. ॥ s'agit de :

- Intégrer le questionnement à toutes les étapes de la démarche (au début, au cours et à la fin d'une investigation);

- Susciter la discussion entre les élèves sur les différentes façons de répondre à une question (protocole) et sur les différentes explications (conclusions) que les élèves proposent à partir d'un résultat issu d'une expérience;

- Susciter la réflexion des élèves sur les limites d'un modèle (exemples du volcan ou du système solaire) pour représenter la réalité;

- Amener les élèves à construire leurs propres modèles (comme le cycle de l'eau) plutôt que de se limiter à la présentation de modèles sur le Web ou sur le tableau numérique interactif;

- Intégrer des démarches d'investigation propres aux sciences et à la technologie dans la réalisation de projets interdisciplinaires (prévoir la réalisation d'expériences, de modèles ou de prototypes dans le cadre d'une recherche);

- Mobiliser différentes formes de langages (croquis, schémas, plans, graphiques) utilisés en sciences et technologie;

- Intégrer de façon plus précise des savoirs (concepts) et des démarches d'investigation (expérimentation, modélisation, 


\section{REVUE HYBRIDE DE L'ÉDUCATION}

conception) dans les planifications et les évaluations des enseignants.

Pour aller plus loin dans ces propositions, nous travaillons maintenant à mettre en forme des exemples de pratique construits avec des enseignants à partir de critères issus des programmes, de la recherche et de la pratique (Couture, Dionne, Savoie-Zajc et Aurousseau 2015), pour fins de diffusion professionnelle et scientifique. Les retombées pour la pratique dépendront alors de l'utilisation qu'en feront les acteurs du milieu scolaire.

Globalement les retombées de nos travaux pour la pratique comprennent:

- Des réinvestissements personnels (plus d'importance accordée au questionnement des élèves et aux démarches d'investigation);

- Des activités et des outils intégrés au répertoire des enseignants (planifications à long terme, trousses, nouvelles activités);

- Des pistes de développement (précision des critères et des objets d'évaluation);

- Des exemples de pratique à diffuser auprès de la communauté de pratique (descriptions appuyées de photos de réalisations d'élèves);

- Des développements de pratiques d'enseignement (ouverture à diverses façons de faire des sciences et de la technologie en classe);

- Élaboration d'un site Web (en construction) pour fins de diffusion. 


\section{REVUE HYBRIDE DE L'ÉDUCATION}

\section{Retombées pour la recherche}

II serait réducteur de mesurer les retombées de travaux de recherche collaborative en nombre de publications et de communications scientifiques. Au-delà de ces productions, le lien théorie-pratique créé par l'ancrage des objets d'étude est une retombée importante. En effet, l'idée de travailler au développement des pratiques d'enseignement va de pair avec le besoin de formation exprimé par les acteurs du milieu. C'est d'ailleurs le point de départ de nos travaux qui illustre bien l'étape de cosituation proposée par Desgagné (1998) en recherche collaborative. Pour la recherche, cette cosituation de l'objet permet de construire des connaissances qui au-delà des propositions théoriques et des prescriptions officielles, intègrent le point de vue de la pratique. En cours de réalisation, dans l'étape de coopération (Desgagné 1998), l'objet se précise passant du développement de la pratique à l'analyse d'ajustements (Savoie-Zajc, 2005) pour ensuite travailler à co-construire ensemble, à l'étape de coproduction (Desgagné, 1998), des exemples de pratique porteurs de retombées pour la pratique, la recherche et la formation. Pour la recherche, l'analyse de tels exemples permet de documenter ce qui se développe collectivement et ce qui peut se faire en classe au regard des prescriptions officielles et des propositions didactiques mises de l'avant par les chercheurs. De cette analyse se dégagent des connaissances co-construites répondant au critère de double vraisemblance (Dubet, 1994) pour la pratique et pour la recherche. En intégrant le point de vue des programmes, des travaux de recherche en didactique et de la pratique, ces connaissances montrent bien le maillage (Proulx, 2013) pouvant se tisser entre recherche et pratique.

En résumé, les retombées de nos travaux pour la recherche se présentent comme suit :

- Un ancrage cositué dans la théorie et la pratique des objets d'étude; 


\section{REVUE HYBRIDE DE L'ÉDUCATION}

- Le développement de connaissances intégrant le point de vue des programmes, des travaux de recherche en didactique et de la pratique;

- Des résultats d'analyse d'exemples de pratique construits avec les enseignants;

- Des productions scientifiques;

- Des développements de pratiques de recherche.

\section{Retombées pour la formation}

En matière de retombées pour la formation, notons que le travail de collaboration que nous menons est source de développement pour tous les acteurs qui y participent, y compris les chercheurs. La rencontre des points de vue provoque des questionnements qui bousculent nos façons de voir l'enseignement de science et technologie. Chacun a alors l'occasion d'intégrer à son cadre de référence des éléments de celui des autres participants. Pour les enseignants, ces questionnements ont surtout porté sur les visées éducatives en science et technologie, leur permettant ainsi de mieux cibler leur priorité. Pour les conseillers pédagogiques, ce sont les possibilités et les contraintes d'application du programme dans la pratique des enseignants, ainsi que l'importance de la planification qui retiennent l'attention. Pour le chercheur, c'est toute la question de la viabilité en contexte (Glaserfeld, 1995) de propositions didactiques qui se pose. De façon plus large, les liens qui s'établissent entre le discours des uns et des autres créent de la cohérence entre les messages véhiculés à l'université et dans le milieu scolaire ce qui, en matière de formation initiale et continue, est souhaitable.

Les retombées potentielles de nos travaux pour la formation sont ainsi envisagées :

- Un meilleur arrimage entre formation initiale et continue;

- L'utilisation des outils et des exemples de pratique construits avec les enseignants en formation initiale et continue; 


\section{REVUE HYBRIDE DE L'ÉDUCATION}

- Des témoignages d'enseignants ayant participé aux projets dans les cours de didactique donnés à l'université;

- Des développements de pratiques de formation initiale et continue.

\section{Pour conclure}

En éducation, la recherche collaborative propose une façon différente de faire de la recherche avec les acteurs du milieu scolaire afin de co-construire des connaissances ayant des doubles retombées pour la pratique et pour la recherche (Bednarz, 2013). C'est dans cette visée que nous travaillons, depuis plus de dix ans, à développer, avec des enseignants et des conseillers pédagogiques du Saguenay, des pratiques d'enseignement de science et technologie. Le défi qui se présente à nous aujourd'hui est celui de la diffusion de ces travaux afin qu'un plus grand nombre d'enseignants puissent s'en inspirer. Des publications professionnelles et scientifiques sont déjà disponibles et un site Web est en construction, mais il y a peut-être autre chose à imaginer ensemble pour un meilleur impact dans la pratique, la recherche et la formation. C'est donc sur une invitation à imaginer ensemble de nouveaux modes de diffusion, respectant les attentes et les contraintes des uns et des autres, que se termine cette réflexion sur les retombées de nos travaux de recherche collaborative portant sur l'enseignement de science et technologie. De notre expérience, nous retenons l'importance de maintenir le dialogue entre la pratique et la recherche dans nos activités de développement, de recherche et de formation. 


\section{REVUE HYBRIDE DE L'ÉDUCATION}

\section{Références}

Bednarz, N. (2013). Regarder ensemble autrement: ancrage et développement des recherches collaboratives en éducation au Québec. Dans N. Bednarz (dir.), La recherche collaborative en action: un autre regard pour la didactique (p. 13-29). Paris, France : L'Harmattan.

Couture, C. (2015). Collaborer pour ajuster les pratiques d'enseignement des sciences et de la technologie à l'élémentaire: vers une didactique plus intégrative. Dans L. G. Samson, N. Sylla et C. Couture (dir.), Le développement professionnel des enseignants de sciences, technologie et mathématique (p. 177-203). Nice, France : Les éditions Ovadia.

Couture, C. (2013). Vers une didactique intégrative: analyse d'ajustements de pratiques d'enseignants du primaire en sciences et technologie. Dans N. Bednarz (dir.), La recherche collaborative en action : un autre regard pour la didactique (p. 309-324). Paris, France : L'Harmattan.

Couture, C. (2010). Accompagner des enseignants pour conjuguer développement pédagogique et didactique des sciences. Dans $\mathrm{C}$. Couture et L. Dionne (dir.), Formation et développement professionnel des enseignants dans le domaine des sciences, de la technologie et des mathématiques : recherches et approches novatrices (p. 131-150). Ottawa, ON : Presses de l'Université d'Ottawa.

Couture, C. (2002). Étude d'un processus de collaboration praticienchercheur pour la coconstruction d'un projet pédagogique en sciences de la nature (Thèse de doctorat non publiée). Université du Québec à Chicoutimi, Université du Québec à Montréal. 


\section{$\&$}

\section{REVUE HYBRIDE DE L'ÉDUCATION}

Couture, C., Dionne, L., Savoie-Zajc, L. et Aurousseau, E. (2015). Le développement des pratiques d'enseignement des sciences et des technologies à l'élémentaire et de recherche en didactique : selon quels critères? Recherches en didactique des sciences et des technologies, 11, 109-132.

Couture, C., Dionne, L., Savoie-Zajc, L. et Aurousseau, E. (2012). Ajustements de pratiques d'enseignants de l'élémentaire en sciences et technologie. Formation et profession: revue scientifique internationale en éducation, 20(3), 1-13.

Desgagné, S., Bednarz, N., Couture, C., Poirier, L. et Lebuis, P. (2001). L'approche collaborative de recherche en éducation : un nouveau rapport à établir entre recherche et formation. Revue des Sciences de l'Éducation, XXVII(1), 33-64.

Desgagné, S. (1998). La position du chercheur en recherche collaborative : illustration de médiation entre culture universitaire et culture scolaire. Recherches qualitatives, 18, 77-105.

Desgagné, S. (1997). Le concept de recherche collaborative : I'idée d'un rapprochement entre chercheurs universitaires et praticiens enseignants. Revue des Sciences de l'Éducation, XXIII(2), 371393.

Dionne, L., Couture, C., Savoie-Zajc, L. et Paris, G. (2014). La communauté d'apprentissage comme expérience vicariante pour rehausser le sentiment d'autoefficacité en sciences d'enseignantes de l'élémentaire. Canadian Journal of Science, Mathematics and Technology Education, 15(1), 15-31.

Dionne, L. et Couture, C. (2013). Avantages et défis d'une communauté d'apprentissage pour dynamiser l'enseignement des sciences et de la technologie à l'élémentaire. Éducation et Francophonie, 41(2), 212-231. 


\section{REVUE HYBRIDE DE L'ÉDUCATION}

Dionne, L., Lemyre, F. et Savoie-Zajc, L. (2010). Vers une définition englobante de la communauté d'apprentissage (ca) comme dispositif de développement professionnel. Revue des sciences de l'éducation, 36(1), 25-43.

Dubet, F. (1994). La sociologie de l'expérience. Paris, France : Éditions du seuil.

Glaserfeld, E.V. (1995). A Constructivist Approach to Teaching. Constructivism in education. New-Jersey : Leslie P. Steffe et Jerry Gale, 3-15.

Hasni, A. et Samson, G. (2011). Pour une collaboration Université-milieu scolaire: des pistes au service de l'apprentissage en sciences et technologie. Québec : Presses de l'Université du Québec.

Kahn, S., Hersant, M. et Orange Ravachol, D. (2010). Savoirs et collaborations entre enseignants et chercheurs en éducation. Recherches en éducation, Hors-série no. 1, Juin.

Legendre, R. (Ed.) (2005). Dictionnaire actuel de l'éducation (3 éd.). Montréal, QC : Guérin.

Martinand, J.-L. (2010). Schémas didactiques pour la modélisation en sciences et technologies. SPECTRE, 4O(1), 20-24.

Martinand, J.-L. (1992). Organisation et mise en œuvre des contenus d'enseignement. Recherches en didactiques: contribution à la formation des maitres. (p. 135-147). Paris, France: Éditions Jacques Colomb.

Ministère de l'Éducation, du Loisir et du Sport (2011). Cadre d'évaluation des apprentissages - Science et technologie - Enseignement primaire $-2^{e}$ et $3^{e}$ cycles. Éducation préscolaire et enseignement primaire. Québec, QC : Gouvernement du Québec. 


\section{$\&$}

\section{REVUE HYBRIDE DE L'ÉDUCATION}

Ministère de l'Éducation du Québec (2001). Le programme de formation de l'école québécoise. Éducation préscolaire et enseignement primaire. Québec, QC : Gouvernement du Québec.

Paillé, P. et Muchielli, A. (2008). L'analyse qualitative en sciences humaines et sociales. Paris, France : Armand Colin.

Proulx, J. (2013). Réflexions épistémologiques sur la recherche collaborative en didactique : possibilités et excès. Dans N. Bednarz (dir.), La recherche collaborative en action : un autre regard pour la didactique (p. 327-351). Paris, France : L'Harmattan.

Samson, G., Couture, C. et Sylla, N. (2015). Recherche participative \& didactique pour les enseignants : Perspectives croisées en Science \& Technologie. Nice, France : Les Éditions Ovadia.

Savoie-Zajc, L. (2011). La recherche qualitative/interprétative en éducation. Dans T. Karsenti, et L. Savoie-Zajc (dir.), La recherche en éducation : étapes et approches (p. 123-147). Saint-Laurent, QC : ERPI.

Savoie-Zajc, L. (2005). Soutenir l'émergence de communauté d'apprentissage au sein de communautés de pratique ou les défis de l'accompagnement au changement. Dans L. Sauvé, I. Orellana et É. Van Steenberghe (dir.), Éducation et environnement: un croisement de savoirs (p. 63-75). Montréal, QC : Les cahiers scientifiques de l'ACFAS.

Savoie-Zajc, L. (2001). La recherche-action en éducation : ses cadres épistémologiques, sa pertinence, ses limites. Dans M. Anadon et M. L'Hostie (dir.), Nouvelles dynamiques de recherche en éducation (p. 15-49). Québec, QC : Les Presses de l'Université Laval. 